\title{
CONTROL ERROR DYNAMIC MODIFICATION AS AN EFFICIENT TOOL FOR REDUCTION OF EFFECTS INTRODUCED BY ACTUATOR CONSTRAINTS
}

\author{
KRZYSZTOF B. JANISZOWSKI \\ Institute of Automatic Control and Robotics \\ Warsaw University of Technology, ul. Św. A. Boboli 8, 02-525 Warsaw, Poland \\ e-mail: kjanisz@mchtr.pw.edu.pl
}

\begin{abstract}
A modification of digital controller algorithms, based on the introduction of a virtual reference value, which never exceeds active constraints in the actuator output is presented and investigated for some algorithms used in single-loop control systems. This idea, derived from virtual modification of a control error, can be used in digital control systems subjected to both magnitude and rate constraints. The modification is introduced in the form of on-line adaptation to the control task. Hence the design of optimal (in a specified sense) digital controller parameters can be separated from actuator constraints. The adaptation of the control algorithm (to actuator constraints) is performed by the transformation of the control error and is equivalent to the introduction of a new, virtual reference value for the control system. An application of this approach is presented through examples of three digital control algorithms: the PID algorithm, the dead-beat controller and the state space controller. In all cases, clear advantages of transients are observed, which yields some general conclusions to the problem of processing actuator constraints in control.
\end{abstract}

Keywords: actuator constraints, digital dead-beat control, PID control, rate constraints, state space control, saturations at control, wind-up.

\section{Introduction}

Constraints of an actuator have always involved many problems in practical application of control techniques. They have affected the basic controller action, prolonged control time and can sometimes induce oscillations that are hard to eliminate. The best known, widely commented on and investigated is the wind-up effect observed in PID control. This commonly used, efficient algorithm suffers from two major drawbacks caused by actuator output constraints: the limitation of the differential action at a rapid variation of the control error and prolonged integration.

The first effect, resulting in less efficient disturbance compensation and a slow following reference signal, can be reduced in part by the introduction of inertia in the differential term. The second effect is more embarrassingthe actuator output saturation yields the integration of the control error in a prolonged time interval. This part of the control action disappears slowly (only by the reduction of the integral action), which yields unwanted, large overshoot-wind-up. A practical approach to the reduction of this effect is either to form another shape of the reference value (e.g., time ramp) or introduce modifica- tions in the integral action of the controller as conditional, reduced or even reversed integration. These methods have been employed to a large extent in digital PID controllers, where modifications of arithmetic processing can be easily introduced in the program.

A saturation of the actuator output has been considered by many authors (Åstrom and Hägglund, 1995; Åstrom and Wittenmark, 1997; Hanus et al., 1987; Kothare et al., 1994; Ngyuen and Jabbari, 2000; Shinskey, 1996). The observed deterioration of the control action, due to a limited actuator output, has forced manufacturers to introduce some alternations of the digital PID algorithm, see, e.g., (Advant®OCS, 1998; Siemens, 1990). The negative effect of the rate constraint in an actuator has also been noticed and some countermeasures have been proposed (Grimm et al., 2003; Hippe, 2003; da Silva et al., 2003; Walgama et al., 1992). Most of these approaches have considered modifications of the PID algorithm, where an integral action has been recognized as the main reason for problems. Serious problems induced by actuator constraints are present in other algorithms, too, e.g., the state space controller working without the integral term (Hippe, 2003; Janiszowski, 2004; Kothare et al., 
1994; Walgama and Sternby, 1993).

The problems caused by actuators have forced researchers to investigate different techniques, which can reduce the observed drawbacks by proper synthesis of controller parameters (Postlethwaite et al., 2003; Hippe, 2003; Kothare et al., 1994; Scottedwarda and Hall, 2001; Walgama et al., 1992). Some of these techniques, like, e.g., the LMI approach (Postlethwaite et al., 2003; Ngyuen and Jabbari, 2000; Turner and Postlethwaite, 2004; Walgama et al., 1992), can even induce the robustness of the control system with respect to varying process properties. In all these cases, design is based on the fundamental assumption: the resulting closed-loop system has to preserve some gain margin for the most unfortunate control case. This assumption usually yields quite conservative design and reduces the dynamics of the closed-loop system. In effect, a digital controller is usually slower than it could be, and less efficient in the most important case - the compensation of disturbances. Simple adaptation of PID algorithms, introduced in industrial solutions, is based on the conditional integration technique (Advant $囚$ OCS, 1998; Åstrom and Wittenmark, 1997; Shinskey, 1996; Siemens, 1990; Walgama et al., 1992), where the integral action of a controller is supervised on-line. A more active approach is presented in the back-up calculation algorithm, where the integral part of the controller output is reduced when the actuator output is saturated (Visioli, 2003).

In all of the above mentioned techniques, countermeasures are used when the activation of actuator constraints is detected by the control system, i.e., the control system is already at the limit of its capabilities and cannot implement its normal function. It is like "symptom healing" and does not remove the real reason: a controller demands values exceeding the interval suitable for the actuator output.

The main aim of the proposed method is based on another approach - the control problem has to be transformed to a form in which the controller output will always be within the active area of the actuator output when necessary at constraints. The application of this approach will be presented through examples of three digital control algorithms: the PID control algorithm, the rarely considered but theoretically fastest dead-beat algorithm, and the phase state space controller.

\section{Problem statement}

Let us consider a set of constraints $\Omega_{v}$ introduced by the digital actuator output $v_{k}$,

$$
\begin{aligned}
\Omega_{v}= & \left\{v_{k}: v_{k} \leq \max v, v_{k} \geq \min v, v_{k}-v_{k-1}\right. \\
& \left.\leq(\max V) \Delta, v_{k}-v_{k-1} \geq-(\min V) \Delta\right\},
\end{aligned}
$$

where the subscript $k$ refers to discrete time instant ( $t=$ $k \Delta, \Delta$ being the sampling interval). Saturation con- straints of the actuator $(\max v, \min v)$ can be different, as well as rate constraints $(\max V$, min $V$ ). A primary reason for the controller output $u_{k}$ exceeding the area $\Omega_{v}$ is always a control error $e_{k}$-the a difference between the reference value $r_{k}$ and the control system output $y_{k}$. It depends on both terms $y_{k}$ and $r_{k}$. The system output $y_{k}$ is fixed at the time instant $k$. However, $r_{k}$ can be substituted by a virtual one $r_{k}^{\prime}$ that prevents the controller output from going beyond $\Omega_{v}$. Let us denote the controller output for some algorithm as a function $R\left(\mathbf{u}_{k-}, e_{k}, \mathbf{e}_{k-}\right)$ of past values of the controller output $\mathbf{u}_{k-}=\left[u_{k-1}, u_{k-2}, \ldots\right]$, the error $e_{k}$ and past values of the control error $\mathbf{e}_{k-}=\left[e_{k-1}, e_{k-2}, \ldots\right]$. We are looking for a modification $r_{k}^{\prime}$ of the actual reference value $r_{k}$ that will hold the controller output within limits introduced by $\Omega_{v}$,

$$
\begin{aligned}
r_{k}^{\prime}= & M\left(u_{k-}, r_{k}, e_{k-}, \Omega_{v}\right) \\
& \Rightarrow u_{k}=R\left(u_{k-}, e_{k}^{\prime}, e_{k-}\right) \in \Omega_{v}, \\
e_{k}^{\prime}= & r_{k}^{\prime}-y_{k} .
\end{aligned}
$$

This reformulation of the problem needs to be completed by extra calculations that preserve a general task of the control system. In the case of calculation units in the equipment used, this extra effort does not exceed computational power. In fact, sometimes this modification is so small that it can be used in simple PLC controllers. A modification based on this principle has already been introduced and tested for the PID algorithm (Janiszowski, 2005), showing good efficiency in both following the reference value and disturbance compensation. In some industrial application areas, e.g., the drive control technique, some functions for reference value pre-forming are incorporated in the controller, and keep the output within the limitations of the electric supply unit. Such a function will be automatically introduced by the suggested modification (2), and will have a form that will always use the full range of actuator capabilities, i.e., it will drive a machine as fast as possible. Now let us consider the proposed approach for three digital control algorithms.

2.1. PID digital control algorithm. A general form of the PID algorithm can be presented by the following relation (̊̊strom and Hägglund, 1995; Shinskey, 1996; Unbehauen, 1987):

$$
M\left(q^{-1}\right) u_{k}=Q\left(q^{-1}\right) r_{k}-P\left(q^{-1}\right) y_{k},
$$

where the polynomials $M\left(q^{-1}\right), Q\left(q^{-1}\right)$ and $P\left(q^{-1}\right)$ can express modifications of the basic PID algorithm, and the operator $q^{-1}$ is a time-shift operator, $v_{k} q^{-p}=v_{k-p}$. The output signal $u_{k}$ of the basic digital PID control closedloop system presented in Fig. 1 contains three forms of processing the control error $e_{k}$ : the proportional action, the derivative action and the integral term. The impact 


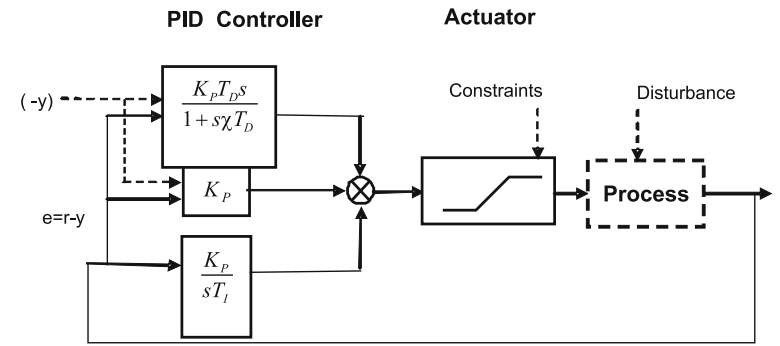

Fig. 1. Structure of the digital control system with the PID algorithm.

of these actions is determined by the algorithm parameters: the gain $K_{p}$, the derivation constant $T_{D}$ and the integration constant $T_{I}$. The controller structure presented in Fig. 1 is widely used for clear definition of all actions and easily implements a bump-less change between the manual and automatic control modes (Advant $\AA$ OCS, 1998; Siemens, 1990). Due to a limitation of the gain of the derivative action, an inertia $T=\kappa \Delta$ is introduced. For the structure presented in Fig. 1, the polynomials (3) can be determined as

$$
\begin{aligned}
P\left(q^{-1}\right) & =Q\left(q^{-1}\right)=p_{0}+p_{1} q^{-1}+p_{2} q^{-2}, \\
\lambda & =\frac{K}{1+\kappa}, \\
p_{0} & =\lambda\left[1+\frac{T_{D}+\kappa \Delta}{\Delta}+\frac{\Delta+\kappa \Delta}{2 T_{I}}\right], \\
p_{1} & =\lambda\left[-1+\frac{\Delta}{2 T_{I}}-2 \frac{\left(T_{D}+\kappa \Delta\right)}{\Delta}\right], \\
p_{2} & =\lambda\left[\frac{T_{D}+\kappa \Delta}{\Delta}-\frac{\kappa \Delta}{2 T_{I}}\right], \\
M\left(q^{-1}\right) & =1+m_{1} q^{-1}+m_{2} q^{-2}, \\
m_{1} & =-\frac{1+2 \kappa}{1+\kappa}, \quad m_{2}=\frac{\kappa}{1+\kappa} .
\end{aligned}
$$

The precise form of (4) depends on the integration formula used a (rectangular or a trapezoidal one), but at a proper value of $\Delta$ the resulting control transients are close. In an ideal PID algorithm, the coefficient $\kappa$ is zero, and the order of polynomial $M\left(q^{-1}\right)$ is reduced to one with $m_{1}=-1$. A different choice of the polynomials $Q\left(q^{-1}\right)$ and $P\left(q^{-1}\right)$ can be used for the reduction of the impact of the derivative action at a step change of the reference value $r_{k}$ (Åstrom and Hägglund, 1995; Unbehauen, 1987).

The controller output for the basic PID algorithm $\left(Q\left(q^{-1}\right)=P\left(q^{-1}\right)\right)$ is determined by the relation

$$
\begin{aligned}
u_{k}= & -m_{1} u_{k-1}-m_{2} u_{k-2}+p_{0} e_{k} \\
& +p_{1} e_{k-1}+p_{2} e_{k-2}
\end{aligned}
$$

that has the form $R\left(\mathbf{u}_{k-}, e_{k}, \mathbf{e}_{k-}\right)$ as mentioned in (2). In an ideal controller ( $T=0)$, Eqn. (5) is simplified to

$$
u_{k}=u_{k-1}+p_{0} e_{k}+p_{1} e_{k-1}+p_{2} e_{k-2} .
$$

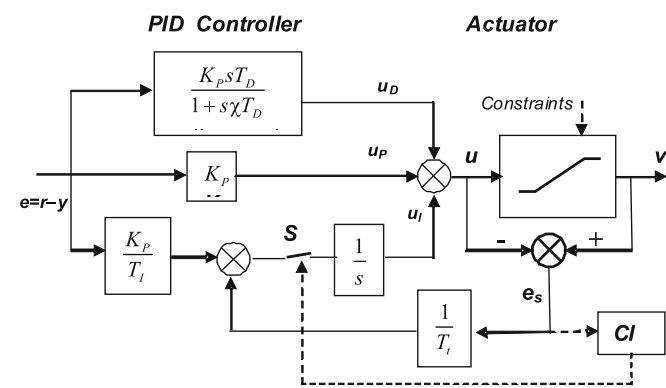

Fig. 2. Structure of the digital control system for the PID algorithm with back-up calculation.

When the controller output value $u_{k}$ lies within $\Omega_{v}$, the reference value $r_{k}$ remains the same. Otherwise, some constraints are met at a minimal (the first condition in (7)) or maximal limit (the second one). The controller output has to be limited if the constraints (1) are to be fullfied,

$$
\begin{aligned}
& v_{+}=\min \left(\max v, u_{k-1}+\Delta \max V\right), \\
& v_{-}=\max \left(\min v, u_{k-1}-\Delta \min V\right) .
\end{aligned}
$$

Now a modification $M(\cdot)$ (2) of the control error $e_{k}$ can be introduced,

$$
r_{k}^{\prime}=\left\{\begin{array}{r}
\frac{1}{p_{0}}\left[v_{+}-u_{k-1}-p_{1} e_{k-1}-p_{2} e_{k-1}\right]+y_{k} \\
\text { if } u_{k} \geq v_{+}, \\
r_{k} \text { if } u_{k} \in \Omega_{v}, \\
\frac{1}{p_{0}}\left[v_{-}-u_{k-1}-p_{1} e_{k-1}-p_{2} e_{k-1}\right]+y_{k} \\
\text { if } u_{k} \leq v_{-} .
\end{array}\right.
$$

All efficient modifications of the PID algorithm have used the constrained output signal (7) as a value $u_{k}$ in the next control steps, and this brings a significant reduction in the wind-up effect. In the suggested modification, the algorithm will use a limited value $u_{k}$, too, but instead of the differences $r_{k-i}-y^{k-i}$, the values $r_{k-i}^{\prime}-y_{k-i}$ will be used. This yields the following PID algorithm:

$$
\begin{aligned}
u_{k} & =u_{k-1}+p_{0} e_{k}^{\prime}+p_{1} e_{k-1}^{\prime}+p_{2} e_{k-2}^{\prime}, \\
e_{k-i}^{\prime} & =r_{k-i}^{\prime}-y_{k-i}, \quad i=0,1,2,
\end{aligned}
$$

which is even more efficient. The integral part of the controller output will now contain a sum of the reduced control error values, and in effect it will not increase as fast as in the classic algorithm (6). Derivative processing of the control error will now be divided into many small steps, forming automatically a ramp-like shape of the reference value. On the other hand, at a significant change of disturbance, this modification of the instant reference value permits a prolonged derivative action of the controller that increases its efficiency in compensation for disturbances. Simulated results for a modified version of the PID algorithm compared with those achieved by other PID algorithms will complete this discussion. 


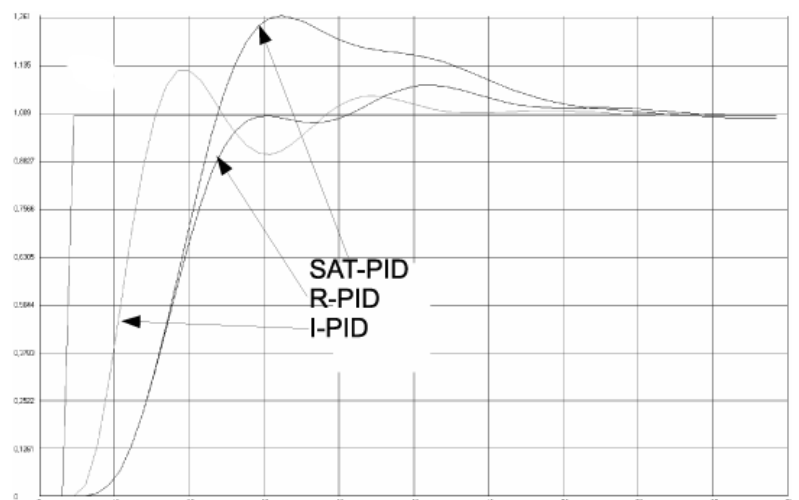

(a)

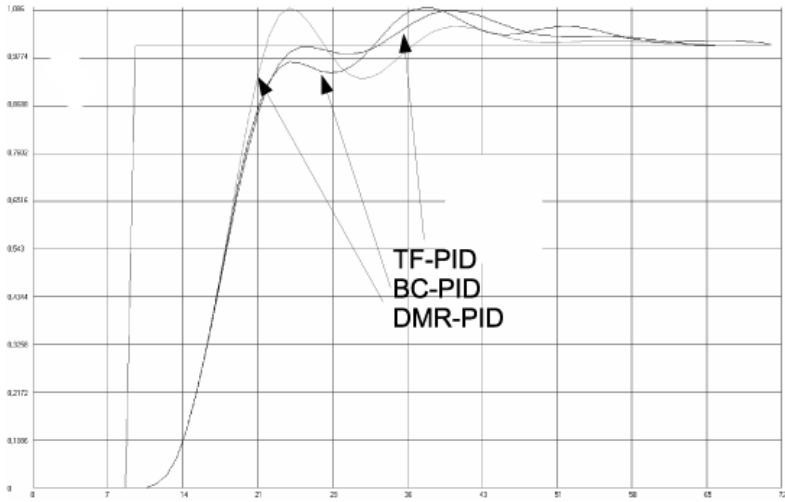

(b)

Fig. 3. Step responses of the closed loop system output for different PID algorithms compared for the process $G_{1}(s)$.

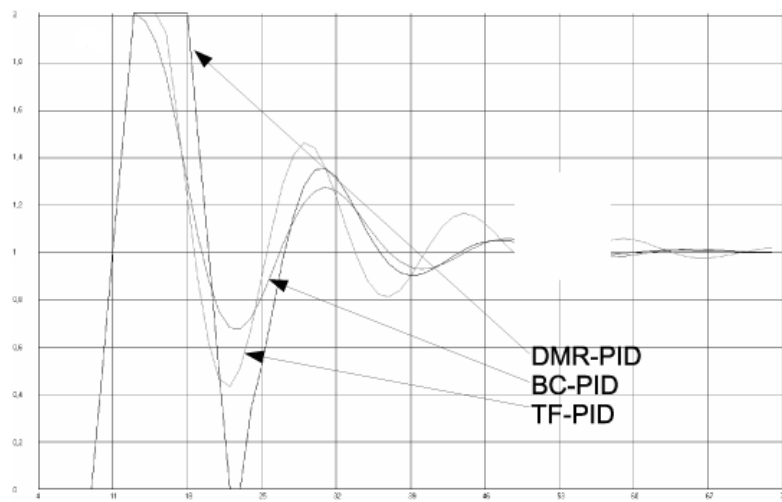

(a)

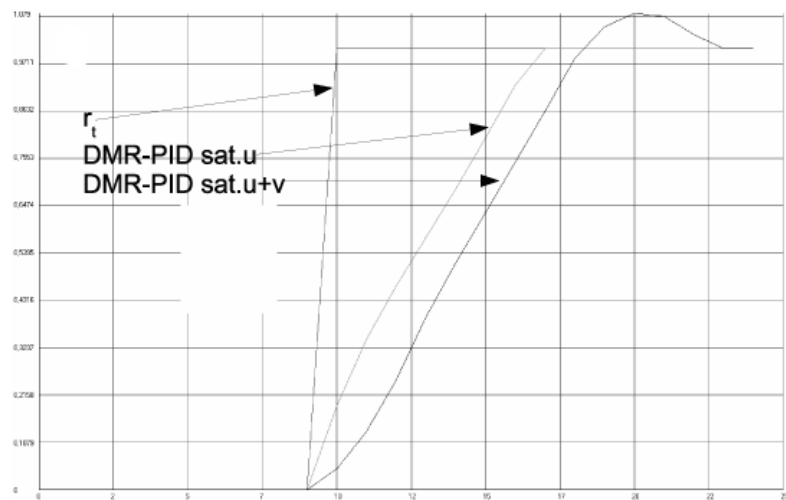

(b)

Fig. 4. (a) Controller output transients for the compared PID algorithms, (b) modified reference value transients for DMR-PIDsat. $u \Leftrightarrow u \in[-2,2]$, sat. $u+v \Leftrightarrow u \in[-2,2], \max V=0.25$.

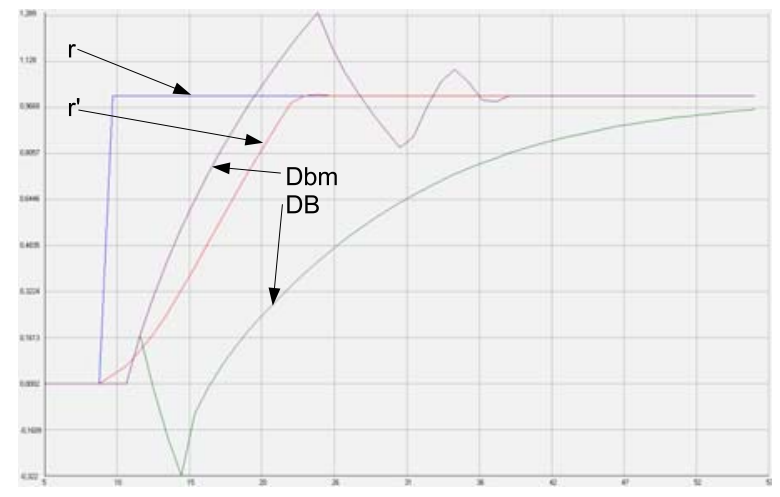

Fig. 5. Transients of the system output for the dead-beat controller and the process $G_{2}(s), r$-the basic reference value, $r^{\prime}$ - the modified reference value trajectory.

As the basic reference, the results achieved by the I-PID algorithm (4) $(\kappa=0)$ will be considered without constraints introduced by the actuator (output or rate). The controller parameters $K, T_{D}, T_{I}$ for the I-PID algorithm were determined for the minimization of the index

$$
I_{R}=\sum_{k=N_{1}}^{N_{2}}\left|r_{k}-y_{k}\right|,
$$

where $N_{1}$ is a discrete time instant of a step change of $r_{k}$ and $\mathrm{N}_{2}$ corresponds to the steady state. The I-PID controller algorithm was determined for a gain margin of 6 dB. A version with no countermeasures, i.e., the PID algorithm (6) but operating with introduced constraints is marked as SAT-PID. A version of the PID algorithm with the inertia $T=\kappa \Delta$ and the integration action stops when the actuator achieves constraints is denoted by R-PID. The value of $\kappa$ is determined as the one yielding the minimum of the index $I_{R}$. Another version of the algorithm (Åstrom and Wittenmark, 1997; Visioli, 2003), called TFPID, is investigated in the form of the relation (3), where the polynomials $M\left(q^{-1}\right), P\left(q^{-1}\right)$ are defined as in (4) but $Q\left(q^{-1}\right)=\rho P\left(q^{-1}\right), \rho \in[0.1,1]$.

The integration action for TF-PID is suspended when saturation of the actuator is achieved and the sign of the control error and the sign of its derivative are the same. Both "free" coefficients $\rho$ and $\kappa$ are tuned for the minimum of $I_{R}$. The most active (in wind-up reduction) is the "back-up calculation" BC-PID algorithm with the structure presented in Fig. 2, where the integral action is decreased by the difference between the actuator output $v$ 


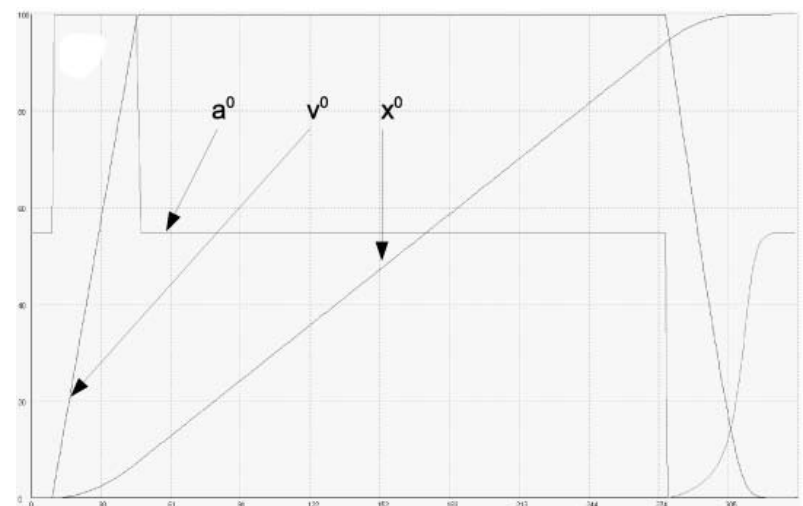

(a)

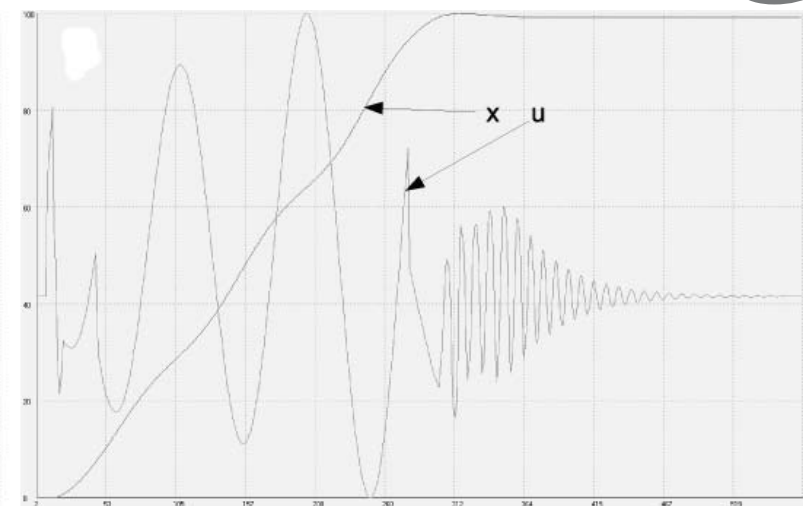

(b)

Fig. 6. (a) Primary required trajectories for the process G3(s), (b) transients of the process output $x$ and the state space controller output $u$ without active output constraints.

and the value of $u$ calculated by the controller,

$$
e_{I}=\frac{K}{T_{I}} e+\frac{1}{T_{t}}(v-u)
$$

The time constant $T_{t}$ in (11) and the inertia $\kappa \Delta$ are determined for the minimum of $I_{R}$. The proposed algorithm (6)-(9) is denoted as DMR-PID (Dynamic Modification of the Reference value). A comparison of these algorithms is made with the use of an example of the SISO system represented by the linear transfer function (Hippe, 2003),

$$
G_{1}(s)=\frac{k}{\left(1+s T_{1}\right)^{3}}, \quad k=1, \quad T_{1}=1 \mathrm{~s} .
$$

For the closed-loop control in (Hippe, 2003), a state space controller was used with the compensator of the control error in the steady state that can cope with windup effects. Numerical results (Hippe, 2003) are comparable (with the transients shown, Fig. 3(b) with respect to the control time, but did not reveal oscillating transients characteristic for PID control. The results gathered in Table 1 were determined with optimized parameters for the I-PID controller: $K=1.89, T_{I}=2.45 \mathrm{~s}, T_{D}=1.12 \mathrm{~s}$, and the sampling interval $\Delta=0.25 \mathrm{~s}$. For a more convenient comparison of results, normalized values of the performance index $I_{\mathrm{XX}-\mathrm{PID}} / I_{\mathrm{I}-\mathrm{PID}}$ are presented in Table 1 . These values were completed with the control time $T_{C}$ and the overshoot $\eta$. The presented results correspond to the cases with output constraint only $v \in[-2,2]$, and with both output and rate constraints $v \in[-2,2]$, $\max V=\min V=0.25$. In the case of magnitude and rate constraints, performance indices are higher and the corresponding transients present longer control time but with less overshoot.

In both cases considered, the suggested DMR-PID algorithm is better than other algorithms in the sense of the index $I_{R}$ and control time $T_{C}$. However, it does not always gives the lowest overshoot (the second case in Table 1). Transients of the step responses for this case are presented in Fig. 3. In Fig. 4(a), controller outputs are plotted for the algorithms TF-PID, BC-PID and DMRPID. DMR-PID has the most efficient action, and has brought the controlled system output to the demanded state in a shorter time. Figure 4(b) presents the values of the virtual reference signal $r_{k}^{\prime}$ generated with (8). In the case of output constraints only (DMR-PID sat. $u$ ), the resulting transient is like a ramp function. For the output and rate constraints (DMR-PID sat. $u+v$ ), this trajectory is smoothed.

2.2. Dead beat control algorithm. This control algorithm theoretically yields the shortest control time in the case of step changes in the reference value. It can be an interesting alternative for fast drive and positioning systems when it is combined with a sufficiently powerful supply unit. The algorithm usually demands a high magnitude of the output, hence it is rarely considered in practice. A modification of its basic action has been proposed (Janiszowski, 1983), but the synthesis needs optimization. In the case of a known transfer function of the controlled SISO linear process $G P\left(q^{-1}\right)$,

$$
G_{P}\left(q^{-1}\right)=\frac{B\left(q^{-1}\right)}{A\left(q^{-1}\right)}=\frac{b_{1} q^{-1}+\cdots+b_{n p} q^{-n p}}{1+a_{1} q^{-1}+\cdots+a_{n p} q^{-n p}},
$$

where the polynomials $B\left(q^{-1}\right)$ and $A\left(q^{-1}\right)$ are co-prime, the dead-beat controller algorithm is determined by a general transfer function (14), where the polynomial $C\left(q^{-1}\right)$ and $V_{0}$, used for the reduction of output magnitude, can be adapted to different tasks (Janiszowski, 1983),

$$
\begin{aligned}
G_{R}\left(q^{-1}\right) & =\frac{u\left(q^{-1}\right)}{e\left(q^{-1}\right)}=\frac{V_{0} A\left(q^{-1}\right) C\left(q^{-1}\right)}{1-V_{0} B\left(q^{-1}\right) C\left(q^{-1}\right)} \\
& =\frac{P\left(q^{-1}\right)}{M\left(q^{-1}\right)}, \\
e_{k} & =r_{k}-y_{k},
\end{aligned}
$$


Table 1. Control results for the process $G_{1}(s)$.

\begin{tabular}{|c|c|c|c|c|c|c|}
\hline Algorithm /Control results & I-PID & SAT-PID & R-PID & TF-PID & BC-PID & DMR-PID \\
\hline & \multicolumn{7}{|c|}{ Actuator constraints: $v \in[-2,2]$} \\
\hline$I_{\text {XX-PID }} / I_{\text {I-PID }}$ & 1 & 3.50 & 1.61 & 1.71 & 1.75 & 1.45 \\
\hline$T_{C}[s]$ & 4.8 & 11.2 & 9.0 & 8.2 & 8.5 & 5.6 \\
\hline$\eta[-]$ & 0.12 & 0.22 & 0.17 & 0.11 & 0.1 & 0.04 \\
\hline & \multicolumn{7}{|c|}{ Actuator constraints: $v \in[-2,2], \max V=\min V=0.25$} \\
\hline$I_{\mathrm{XX}-\mathrm{PID}} / I_{\mathrm{I}-\mathrm{PID}}$ & 1 & 2.81 & 2.12 & 2.08 & 2.10 & 2.0 \\
\hline$T_{C}[s]$ & 4.8 & 10.8 & 9.7 & 8.0 & 8.3 & 6.8 \\
\hline$\eta[-]$ & 0.12 & 0.26 & 0.06 & 0.08 & 0.09 & 0.08 \\
\hline
\end{tabular}

$$
\begin{aligned}
C\left(q^{-1}\right) & =1+c_{1} q^{-1}+\cdots+c_{n c} q^{-n c}, \\
c_{i} & \geq 0, \quad i=0, \ldots, n c \\
V_{0} & =\left[\left(\sum_{i=0}^{n p} b_{i}\right)\left(\sum_{i=0}^{n c} c_{i}\right)\right]^{-1} .
\end{aligned}
$$

For any stable, stationary plant $G P\left(q^{-1}\right)$, a formula for the controller output calculation is (5)

$$
u_{k}=-\sum_{i=1}^{n} m_{i} u_{k-i}+\sum_{i=0}^{n} p_{i} e_{k-i}
$$

where the order of the controller $n$ is equal to $n_{P}+n_{C}$. At the activation of the constraints $\Omega_{v}$, a modification, corresponding to (8), can be introduced and the controller follows a new virtual reference value $r_{k}^{\prime}$. This modified reference value will have the form adapted to actuator capabilities.

Let us consider an SISO plant represented by the transfer function $G_{2}(s)$,

$$
\begin{aligned}
G_{2}(s)= & \frac{\left(1+13.75 s+62.5 s^{2}\right) e^{-s T_{0}}}{\left(1+s T_{1}\right)\left(1+s T_{2}\right)\left(1+s T_{3}\right)} \\
& \Leftrightarrow T_{0}=4 s, \quad T_{1}=5 \mathrm{~s}, \quad T_{2}=10 \mathrm{~s}, \\
G_{2}\left(q^{-1}\right)= & \frac{0.084 q^{-3}-0.1313 q^{-4}+0.0523 q^{-5}}{1-2.4 q^{-1}+1.905 q^{-2}-0.5 q^{-3}}, \\
T_{3}= & 20 \mathrm{~s},
\end{aligned}
$$

with the discrete-time representation $G 2\left(q^{-1}\right)$ (for the sampling interval $\Delta=2 s)$. The value $V_{0}$ (for $C\left(q^{-1}\right)=$ 1 ) is equal to the magnitude of the controller output for the step of $r_{k}$. For the process $G 2\left(q^{-1}\right)$, this value is higher than 200.

Let us consider a limitation of the actuator output $v \in[-2,2]$. The application of the modification with the polynomial $C\left(q^{-1}\right)=1+1,6 q^{-1}+0.6 q^{-2}$ will reduce the actuator output by the factor $3.2(1+1.6+0.6)$, and it will prolong the control time only by $2 \Delta$, up to $7 \Delta=14 \mathrm{~s}$. This reduction does not allow us to apply to the controller its dead-beat control for the actuator output $v \in[-2,2]$, see Fig. 5 (DB). The results of control with the application of (7) and (8), generating the virtual reference value $r_{k}^{\prime}$, are marked as DBm in Fig. 5. The modified control algorithm $\mathrm{DBm}$ follows quite a different reference value $r_{k}^{\prime}$ and realizes dead-beat control. The control time is now prolonged to a time instant when the reference value $r_{k}^{\prime}$ has achieved a steady value, plus the interval of $14 \mathrm{~s}$ resulting from the controller order. The transients presented in Fig. 5 are different from the ones usually achieved by PID control. For the dead-beat control algorithm, the most important is very short control time. The acceptance of the transients presented in Fig. 5 is a problem of application, but the overshoot $\eta=0.23$ is comparable with very dynamic PID control behavior.

\section{Phase state-space controller}

The applications of the above-presented approach are used in relatively easy problems: to drive an output of the controlled system to a steady state with the modification of the virtual reference value. In the case of a predefined trajectory, e.g., for the positioning drive system that should be moved out from one position to another, new problems can arise. A variation of the reference value for velocity could bring an error in the final position. An application of the proposed approach to the state space controller will present that problem.

Different practical problems in the realization of positioning control with pneumatic cylinders controlled by proportional servo-valves and state space control algorithms were presented in (Janiszowski, 2004). The mechanical structure of such a system - a light, powerful pneumatic cylinder, combined with a servo-valve and a linear measurement unit-has created an attractive construction. A model of the valve-cylinder unit can be described as a dynamic process (Janiszowski, 2004),

$$
G_{x u}(s)=\frac{C \omega_{0}^{2}}{s\left(s^{2}+2 \varsigma \omega_{0} s+\omega_{0}^{2}\right)} e^{-s \tau},
$$

where $x$ is the piston position, $u$ is the voltage input to the servo-valve, $C$ is the velocity gain, $\omega$ is natural frequency, $\zeta$ is the dumping factor, and $\tau$ is a delay caused by the transfer of compressed air to cylinder volumes. The control of the above process seems easy, e.g., with the application of a properly designed pole-placement controller, 
but the model (18) strongly depends on the piston velocity. The identification of the Takagi-Sugeno fuzzy model (18) shows (Janiszowski, 2004) a wide range of parameter variations Table 2. A strong influence of the piston velocity on dynamic parameters of the valve-cylinder model comes from the impact of the nonlinear friction force.

The presented variation in the model parameters reflects the basic problem of controller design: for movement with high velocity (the main phase of each movement), the control alghorithm has to correspond to different dynamics than in the last phase of each movement (precise braking) when fine control of a piston without overshoot is necessary. The application of the introduced approach will be presented with an example of positioning control for the fuzzy model (18) of the process with parameters variation as in Table 2 .

A state space controller is defined in classical form:

$$
u_{k}=K_{X}\left[x_{k}^{0}-x_{k}\right]-K_{V}\left[v_{k}^{0}-v_{k}\right]-K_{A}\left[a_{k}^{0}-a_{k}\right] \text {, }
$$

where $x_{k}, v_{k}, a_{k}$ are phase state variables denoting position, velocity and acceleration, respectively, and $x_{k}^{0}, v_{k}^{0}, a_{k}^{0}$ are required trajectories defined in a coherent way, i.e.

$$
v_{k}^{0}=\int_{\tau=0}^{\tau=k \Delta} a^{0}(\tau) \mathrm{d} \tau, \quad x_{k}^{0}=\int_{\tau=0}^{\tau=k \Delta} v^{0}(\tau) \mathrm{d} \tau .
$$

The selection of the controller gains $K_{X}, K_{V}$ and $K_{A}$ is determined for the velocity trajectory (in a trapezoidal form) and the least overshoot at the braking phase of displacement. Scaled trajectories are shown in Fig. 6(a). The velocity $v_{\text {mean }}=1.5 \mathrm{~m} / \mathrm{s}$ achieved after primary acceleration of $\sim 4 g$ is reduced to 0 with deceleration of $\sim 5 g$. The transients of the resulting displacement with an optimaly tuned state-space controller are presented in Fig. 6(b). Positioning control is accurate (overshoot is less than $0.6 \mathrm{~mm}$ ) but demands controller output magnitude of approximately $97 \mathrm{~V}$, i.e., 10 times higher than it is available in the control system (10 $\mathrm{V}$ is the maximal input to the valve). The velocity transients presented in Fig. 6(b) show nonstable behavior of piston velocity at the phase of fast displacement (too large controller gains). For the breaking phase of positioning, the control is weakly dumped but quite accurate and fast. High gains in the state controller system are necessary to prevent the existence of the overshoot at the braking phase (Janiszowski, 2003). The limitation of the valve response to the magnitude of $10 \mathrm{~V}$ and the rate value of $10 \mathrm{~V} / \mathrm{ms}$ results in nonstable positioning transients, Fig. 7(a).

The suggested modification of the reference value was introduced for the control law (19). When the value (19) lies within constraints determined by $\Omega_{v}$, such control is set to the actuator (proportional electropneumatic valve). When the calculated controller output has achieved its lower or upper limits, $v_{-}$or $v_{+}$, respectively, cf. (7), a modification had to be introduced. A modified trajectory of positioning has to be generated in such way so as to satisfy the following conditions:

(a) a new set of trajectories $x_{k}^{\prime 0}, v_{k}^{\prime 0}, a_{k}^{\prime 0}$ is coherent (20) and has to fulfill boundary conditions, i.e., $x_{N}^{\prime 0}=$ $x_{f}=0.4 \mathrm{~m}, v_{\text {mean }}=1.5 \mathrm{~m} / \mathrm{s}$, where $N$ is corresponding stop instant,

(b) the controller output is determined with (19) and the coefficients $K_{X}, K_{V}$ and $K_{A}$,

(c) the value of $u_{k}$ resulting from (19) has to be equal to $v_{-}$or $v_{+}$.

The above conditions are satisfied by the following algorithm:

Step 1. In each step of the constrained control, a set of coherent trajectories $T\left(x_{a}^{\prime 0}, v_{a}^{\prime 0}, a^{\prime 0}, a_{\text {max }}, v_{\text {mean }}, x_{f}\right)$ was generated with the trapezoidal form of velocity for $v_{\text {mean }}=1.5 \mathrm{~m} / \mathrm{s}$.

Step 2. If

$$
u_{a}=K_{X}\left[x_{k}^{\prime 0}-x_{k}\right]+K_{V}\left[v_{k}^{\prime 0}-v_{k}\right]+K_{A}\left[a_{k}^{\prime 0}-a_{k}\right]
$$

is in the area $\Omega_{v}$, this control is set to the valve, otherwise a correction $\Delta a_{k}$ of the acceleration is introduced. This correction $\Delta a_{k}$ has to satisfy conditions that are derived from the bounds (20),

$$
\begin{aligned}
& a_{k}^{\prime 0}=a_{k}+\Delta a_{k}, \\
& v_{k}^{\prime 0}=v_{k}+\Delta\left(a_{k}+\Delta a_{k}\right), \\
& x_{k}^{\prime 0}=x_{k}+0.5 \Delta\left[2 v_{k}+\Delta\left(a_{k}+\Delta a_{k}\right)\right],
\end{aligned}
$$

and, finally, the extreme, acceptable value of the acceleration correction $\Delta a_{k}$ is

$$
\Delta a_{k}=\frac{u_{k}^{*}-\Delta\left[K_{X} v_{k}+0.5 \Delta a_{k}\right]-0.5 \Delta^{2} K_{V} v_{k}}{0.5 \Delta^{2} K_{X}+\Delta K_{V}+K_{A}} .
$$

The limited value $u_{k}$ has to be equal to $u_{k}^{*}=v_{-}$ or $v_{+}$and has to fulfill the control law (19). The proposed procedure requires in each step the determination of the modified displacement trajectory $T$ and the correction (23). This extra calculation effort, although higher than in the case of simple PID or dead-beat algorithms, can be realized on-line. The modified displacement trajectory $x_{k}^{\prime 0}$ is changed almost at each step, see Fig. 7(b). It is very close to the trajectory shown in Fig. 7(a), but there some peaks are visible where the controller output is not limited by constraints and can follow the trajectory modified in previous steps. The whole displacement is stable and implemented within $370 \mathrm{~ms}$, including the overshoot in the range of $9.7 \mathrm{~mm}$. Testing this control on a real stand has shown that the overshoot was reduced to $0.7 \mathrm{~mm}$ by the adhesive friction force not included in the model (18). 
Table 2. Variation of parameters $C, \omega 0, \zeta$ of the model (18).

\begin{tabular}{|c|c|c|c|}
\hline $\begin{array}{c}\text { Piston velocity } \mid v \\
{[\mathrm{~m} / \mathrm{s}]}\end{array}$ & $\begin{array}{c}\text { Velocity gain } C \\
{[(\mathrm{~m} / \mathrm{s}) / \mathrm{V}]}\end{array}$ & $\begin{array}{c}\text { Natural frequency } \omega_{0} \\
{[\mathrm{rd} / \mathrm{s}]}\end{array}$ & $\begin{array}{c}\text { Dumping factor } \zeta \\
{[-]}\end{array}$ \\
\hline$[0,0.025]$ & 0.008 & 181 & 7.6 \\
\hline$[0.04,0.1]$ & 0.063 & 83.7 & 1.15 \\
\hline$[0.12,0.25]$ & 0.617 & 34.9 & 0.61 \\
\hline$[0.3,2]$ & 1.545 & 18.5 & 0.23 \\
\hline
\end{tabular}

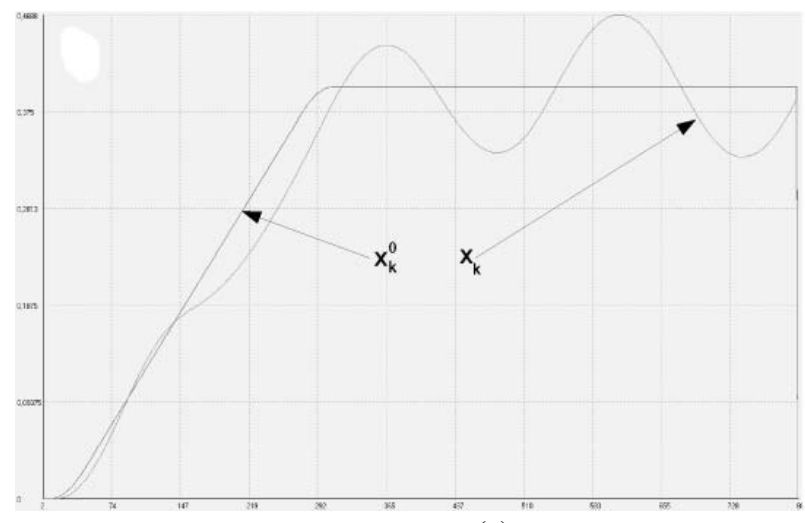

(a)

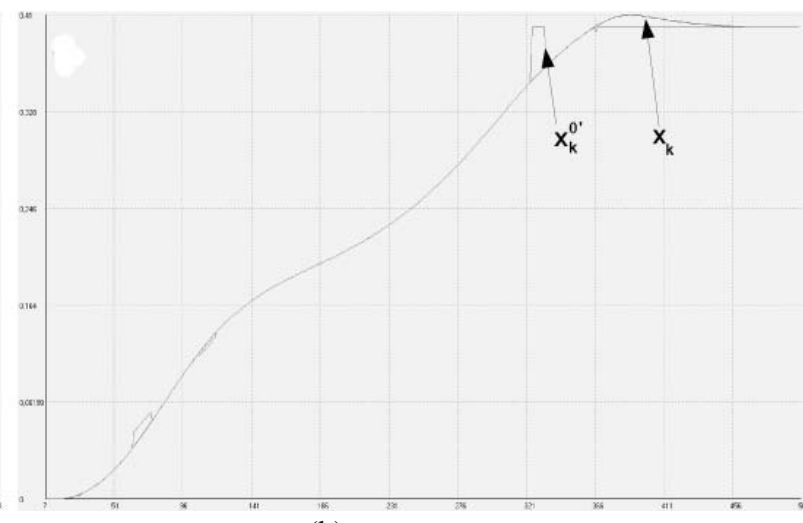

(b)

Fig. 7. (a) Required trajectory $x_{k}^{0}$ and the realized piston displacement $x_{k}$ in the case of the saturated valve output and state space control, (b) the piston displacement $x_{k}$ and the modified trajectory $x_{k}^{0^{\prime}}$.

The most important, however, is stable behavior of the controller which is not forced to behave as in Fig. 6(a). The modified trajectory (22) is always in the area of the capability of the control valve.

\section{Conclusions}

The approach to control error dynamic modification that maintains the activity of a control system for the whole control time has been proposed. Simple rules for the modification together with a relatively flexible definition of constraints introduced by the actuator (1) can be used for different control algorithms and seem quite useful. In the case of steady state control, as for PID and dead-beat controllers, the approach can be used with negligible additional calculation effort. More advanced problems, like, e.g., following some trajectory, demand more calculations but this effort is not excessive and can be performed online.

The separation of control modification from controller design is a clear advantage of the proposed approach. Controller parameters optimized for a specific performance index can be used without the necessity for adaptation when actuator constraints are changed. The controller output is always adapted to the full power of the actuator (7), and a successive modification of the virtual reference value is always optimal in the sense of the least control time.

The suggested approach (DMR-PID control) preserved the full power in the case of disturbance compensation because the derivative action is not suppressed as in all other modifications - R-PID, TF-PID or BC-PID - that apply inertia in the derivative term. The efficient control is combined with more comfort at the design stage. The controller can be tuned with the least reasonable sampling interval (nowadays, this interval is adapted to the actuator

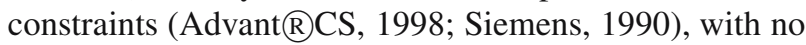
constraints considered at the design phase. The problem of controller parameters tuning with a respectable gain or phase margin still exists but is not affected by saturation or rate constraints in the actuator, which is often the main problem of design. The modification will automatically adapt the control to actuator constraints with small additional calculation effort. It will be interesting to check how the approach will behave in the case of predictive control-the modification could exclude the confusing and time consuming problem of nonlinear optimization in the case of the multi-step controller output trajectory.

\section{References}

Advant $\AA \mathrm{CS}$ (1998). User's Guide, ABB Industrial Systems AB.

Åstrom, K. and Hägglund, T. (1995). PID Controllers, Theory, Design and Tuning, 2nd Edition, ISA Press, London.

Åstrom, K. and Wittenmark, B. (1997). Computer Controlled Systems-Theory and Design, Prentice Hall, New York, NY.

Grimm, G., Postlethwaite, I., Teel, A. T., Turner, M. C. and Zaccarian, L. (2003). Case studies using linear matrix inequalities for optimal anti-windup synthesis, European Journal of Control 9: 463-470. 
Hanus, R., Kinnaert, M. and Henrotte, J. L. (1987). Conditioning technique, a general anti-windup and bumpless transfer method, Automatica 23: 729-739.

Hippe, P. (2003). Windup prevention for unstable systems, $A u$ tomatica 39: 1967-1973.

Janiszowski, K. (1983). A linear digital controller for single loop control systems, International Journal on Control 37(1): 159-174.

Janiszowski, K. (2004). Adaptation, modelling of dynamic drives and controller design in servomechanism pneumatic systems, IEEE Proceedings-Control Theory \& Applications 151(2): 234-245.

Janiszowski, K. (2005). Dynamic modification of reference value for improvement of PID control, Proceedings of the IEEE Conference on Methods and Models in Automation and Robotics, MMAR, Międzyzdroje, Poland, (on CD-ROM).

Kothare, M. V., Campo, P. J., Morari, M. and Nett, C.N. (1994). A unified framework for the study of anti-windup designs, Automatica 30(12): 1869-1883.

Ngyuen, T. and Jabbari, F. (2000). Output feedback controllers for disturbance attenuation with actuator amplitude and rate saturation, Automatica 36: 1339-1346.

Shinskey, F.G. (1996). Process Control Systems-Application, Design, Tuning, Mc-Graw Hill, New York, NY.

Siemens (1990). Compact Controller SIPART DR20, Project planning manual.

da Silva, J. M. G., Tarbouriech, S. and Garcia, G. (2003). Local stabilisation of linear systems under amplitude and rate saturating actuators, IEEE Transactions on Automatic Control 48(5): 842-847.

Scottedward, H. A. and Hall, C. E. (2001). Variable structure PID control to prevent integrator windup, IEEE Transactions on Electronics 48: 442-451.
Turner, M. C. and Postlethwaite, I. (2004). A new perspective on static and low order anti-windup synthesis, International Journal on Control 77(1): 27-44.

Unbehauen, H. (1987). Regelungstechnik II, Vieweg Verlag, Braunschweig.

Visioli, A. (2003). Modified anti-windup scheme for PID controllers, IEE Proceedings-D 150(1): 49-54.

Walgama, K. S., Rönback, S. and Sternby, J. (1992). Generalisation of conditioning technique for anti-windup compensators, IEE Proceedings - D 132(2): 705-724.

Walgama, K. S. and Sternby J. (1993). On the convergence properties of adaptive pole-placement controllers with antiwindup compensators, IEEE Transactions on Automatic Control 38(1): 128-132.

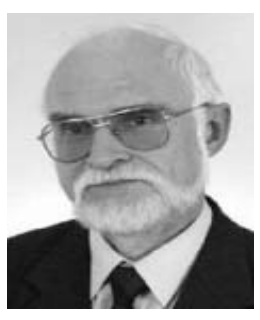

Krzysztof B. Janiszowski is affiliated with the Institute of Automatics and Robotics of the Warsaw University of Technology and is the head of the Department of Actuators for Automatics and Robotics. The areas of his activity include the modelling and identification of dynamic systems, the control of industrial processes, and modern control in servo-systems. His works have been applied to, for example, a new generation of fast pneumatic servo-drives (produced by FESTO), the support of an operator training and decision system for the control of pirolytic ovens in the newly built installation Olefiny II in Płock, Poland, a prototype of a fast hydro-static drive for a new generation of high-speed moulding machines. He is the author of over 130 papers published in periodicals and at scientific conferences.

Received: 22 July 2008

Revised: 10 October 2008 